\title{
Phenomenological study of the double radiative decay $B \rightarrow K \gamma \gamma$
}

\author{
Gudrun Hiller \\ Institut für Physik, Universität Dortmund, D-44221 Dortmund, Germany \\ E-mail: ghillerephysik.uni-dortmund.de \\ A. Salim Safir* \\ CERN, Department of Physics, Theory Unit, CH-1211 Geneva 23, Switzerland \\ E-mail: safir@mail.cern.ch
}

\begin{abstract}
Using the operator product expansion (OPE) technique, we study the rare double radiative decay $B \rightarrow K \gamma \gamma$ in the Standard Model (SM) and beyond. We estimate the short-distance (SD) contribution to the decay amplitude in a region of the phase space which is around the point where all decay products have energy $\sim m_{b} / 3$ in the rest frame of the $B$-meson. At lowest order in $1 / m_{b}$, the $B \rightarrow K \gamma \gamma$ matrix element is then expressed in terms of the usual $B \rightarrow K$ form factors known from semileptonic rare decays. The integrated SD branching ratio in the SM in the OPE region turns out to be $\triangle \mathscr{B}(B \rightarrow K \gamma \gamma)_{S M}^{O P E} \simeq 1 \times 10^{-9}$. We work out the di-photon invariant mass distribution with and without the resonant background through $B \rightarrow K\left\{\eta_{c}, \chi_{c 0}\right\} \rightarrow K \gamma \gamma$. In the SM, the resonance contribution is dominant in the region of phase space where the OPE is valid. On the other hand, the present experimental upper limit on $B_{S} \rightarrow \tau^{+} \tau^{-}$decays, leaves considerable room for New Physics (NP) in the one-particle-irreducible contribution to $B \rightarrow K \gamma \gamma$ decays. In this case, we find that the SD $B \rightarrow K \gamma \gamma$ branching ratio can be enhanced by one order of magnitude with respect to its SM value and the SD contribution can lie outside of the resonance peaks.
\end{abstract}

International Europhysics Conference on High Energy Physics

July 21st - 27th 2005

Lisboa, Portugal

* Speaker. 
Flavor changing neutral currents (FCNC) $B$-decays are of utmost importance in exploring and shaping the flavor structure of the SM and its extensions, since they are forbidden in the Born approximation and highly sensitive to NP contributions in the loops. Although many studies, either experimentally or theoretically, have been devoted to $b \rightarrow s \gamma$ and $b \rightarrow s \ell^{+} \ell^{-}$transitions, less attention has been paid to the double-photon decays mediated by $b \rightarrow s \gamma \gamma$. At the quark level it receives one-particle-reducible (1PR) contributions from the $b \rightarrow s \gamma$ transition plus an additional photon and a one-particle-irreducible (1PI) term from a fermion loop with the two photons emitted from that loop (see Fig 1). At the lowest order, the corresponding amplitude can be written as $[1,2]$

$$
\mathscr{A}(b \rightarrow s \gamma \gamma)=-\frac{i e^{2} G_{F}}{\sqrt{2} \pi^{2}} \lambda_{t} \bar{u}_{s}\left(p^{\prime}\right) \cdot\left[\frac{4}{9}\left(3 C_{1}+C_{2}\right) \kappa_{c} W_{2}^{\mu \nu}-\frac{1}{3} C_{7} W_{7}^{\mu \nu}\right] \cdot u_{b}(p) \varepsilon_{\mu}\left(k_{1}\right) \varepsilon_{v}\left(k_{2}\right),
$$

where $\lambda_{t}=V_{t b} V_{t s}^{*}$ and $p\left(p^{\prime}\right)$ represents the momentum of the $b(s)$-quark. We denote by $k_{1}, k_{2}$ the 4-momenta and by $\varepsilon\left(k_{1,2}\right)$ the polarization vectors of the photons. The tensors $W_{2}^{\mu v}$ and $W_{7}^{\mu v}$ exhibit the contribution of the four-fermion operators $\mathscr{O}_{1,2} \simeq\left(\bar{s} \gamma_{\mu} L c\right)\left(\bar{c} \gamma^{\mu} L b\right)$ and the photon dipole operator $\mathscr{O}_{7} \sim \bar{s} \sigma_{\mu v} F^{\mu v} R b$, respectively, and are given together with the loop function $\kappa_{c}$ in [1].

In this work we consider the exclusive $B \rightarrow K \gamma \gamma$ decay. Since its matrix element induced by $\mathscr{O}_{7}$ is non-local, it is difficult to estimate model-independently. Previous works [3,4] used here solely vector meson dominance models. Instead, we use OPE techniques, which allow to $i$ make the matrix element local in a specific kinematic region by choosing appropriate photon energies and then $i i$ express the matrix element in terms of known form factors. Specifically, we demand both the internal $s$ - and $b$-quarks to be far off-shell, with virtualities of order $m_{b}$. For example, if both photons have energies $m_{b} / 3$, then the intermediate propagators of the 1PR diagrams $\left(Q_{1,2}^{s}\right)^{2}=$ $\left(p^{\prime}+k_{2,1}\right)^{2}$ and $\left(Q_{1,2}^{b}\right)^{2}=-\left(\left(p-k_{1,2}\right)^{2}-m_{b}^{2}\right)$ equal $m_{b} / \sqrt{3}$ and $m_{b} \sqrt{2 / 3}$, respectively. Then we integrate out large scales of order $m_{b}$. We construct the vertices in the effective theory out of a bottom heavy HQET quark $h_{v}$ and a strange collinear SCET quark $\chi[5,6]$. Here, $v=p_{B} / m_{B}$ and a light-like vector $n=p_{K} / E_{K}$, where $E_{K}, p_{K}$ denotes the energy, 4-momentum of the kaon. Hence, we perform an OPE in $\Lambda_{Q C D} / Q$, where $Q=\left\{m_{b}, E_{K}, Q_{1,2}^{s, b}, \sqrt{q^{2}}\right\}$ and $q^{2}=\left(k_{1}+k_{2}\right)^{2}$.

For the lowest order matching onto the $b \rightarrow s \gamma \gamma$ amplitude, we obtain after using the equations of motion the following dimension 8 operators [1]

$$
\frac{m_{b}}{4} \bar{\chi} \sigma_{\mu v} \sigma_{\alpha \beta} R h_{v} F_{1}^{\alpha \beta} F_{2}^{\mu v}, \quad-2 i m_{b} \bar{\chi} \sigma_{\mu v} R h_{v} F_{1}^{\mu v} F_{2}^{\alpha \beta} v^{\alpha} n^{\beta}, \quad \bar{\chi} \gamma^{\mu} L h_{v} F_{1}^{\alpha \beta} D_{\alpha} \tilde{F}_{2 \beta \mu} \quad+(1 \leftrightarrow 2)
$$

where $\tilde{F}_{\mu \nu}=1 / 2 \varepsilon_{\mu v \alpha \beta} F^{\alpha \beta}$ and Wilson lines are understood in $\chi$. The $B \rightarrow K \gamma \gamma$ matrix elements are then obtained from tree level matching of the QCD onto the SCET currents $[5,7,8]$ as

$$
\begin{aligned}
\left\langle K(n)\left|\bar{\chi} h_{v}\right| B(v)\right\rangle & =2 E_{K} \zeta\left(E_{K}\right), & \left\langle K(n)\left|\bar{\chi} \sigma_{\mu v} h_{v}\right| B(v)\right\rangle & =-2 i E_{K} \zeta\left(E_{K}\right)\left(v_{\mu} n_{v}-v_{v} n_{\mu}\right), \\
\left\langle K(n)\left|\bar{\chi} \gamma_{\mu} h_{v}\right| B(v)\right\rangle & =2 E_{K} \zeta\left(E_{K}\right) n_{\mu}, & \left\langle K(n)\left|\bar{\chi} \sigma_{\mu v} \gamma_{5} h_{v}\right| B(v)\right\rangle & =-2 E_{K} \zeta\left(E_{K}\right) \varepsilon_{\mu v \alpha \beta} v^{\alpha} n^{\beta},
\end{aligned}
$$

where the form factor $\zeta\left(E_{K}\right)$ can be identified with the QCD form factor in the usual parametrization, as $\zeta=f_{+}$. It is the only form factor remaining in the symmetry limit, known e.g. from studies in $B \rightarrow K \ell^{+} \ell^{-}$decays. Analytical formulae for the $B \rightarrow K \gamma \gamma$ amplitude from the OPE are given in [1].

An additional contribution to $B \rightarrow K \gamma \gamma$ beyond the OPE is photon radiation off the spectator. It is either kinematically excluded (soft gluons between active quarks and spectator) or suppressed 

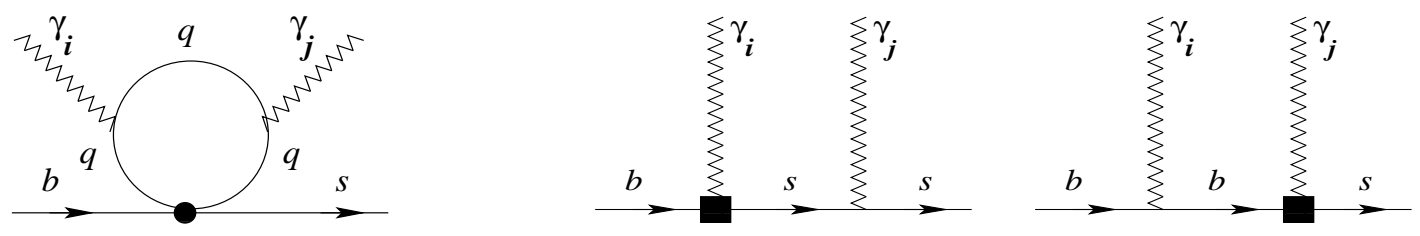

Figure 1: Leading order Feynman diagrams for $b \rightarrow s \gamma \gamma$ decays. Left plot: 1PI diagram from four-fermion operators, other two plots: insertion of $\mathrm{O}_{7}$. Figure taken from [1].

by $\alpha_{s}$. Annihilation contributions are leading power in $1 / Q$, but suppressed by CKM elements or small penguin Wilson coefficients (for further details, see [1]).

Long-distance effects from $B \rightarrow\left(\eta_{x} \rightarrow \gamma \gamma\right) K$, where $\eta_{x}=\eta, \eta^{\prime}, \eta_{c}$ and $B \rightarrow\left(K^{*} \rightarrow K \gamma\right) \gamma$ are sizeable in $B \rightarrow K \gamma \gamma$ decays [3,4]. Both the $K^{*}$ - and the $\eta^{(\prime)}$-channels require kinematics that is outside of our OPE window. Therefore, we take in our analysis only the charmonia, specifically the $\eta_{c}$ and also the $\chi_{c 0}$, into account. (The current upper bound on $\mathscr{B}\left(B \rightarrow \chi_{c 2} K\right)$ implies that the background from the $\chi_{c 2}$ is at least one order of magnitude down with respect to the scalar one.) We estimate the long-distance contamination from $\eta_{c}, \chi_{c 0}$ with a Breit-Wigner ansatz fitted to data [1].

The resulting $B \rightarrow K \gamma \gamma$ di-photon mass spectra $d \Gamma / d q^{2}$ are shown in Fig. 2. (For cuts and input, see [1]). The SD part of the SM spectrum (solid curve) is completely hidden behind the $\eta_{c}$ and the $\chi_{c 0}$ resonance contribution (dash-dotted curve). The integrated SD branching ratio in the SM with cuts is small, $\triangle \mathscr{B}(B \rightarrow K \gamma \gamma)_{S M}^{O P E} \simeq 1 \times 10^{-9}$, with about $50 \%$ uncertainty from the renormalization scale. The contribution from 1PI SD diagrams only gives a somewhat reduced branching ratio $\mathscr{B}(B \rightarrow K \gamma \gamma)_{S M}^{O P E 1 P I} \simeq 0.5 \times 10^{-9}$. Such small SD branching ratios for double radiative decays are not a complete surprise. In fact, being of the same order in $\alpha_{e m}$ as semileptonic rare decays with branching ratios of few $\times 10^{-7}$ [9], we expect with $C_{2} \simeq 1.1$ and $C_{7}^{S M} \simeq-0.3$

$$
\mathscr{B}(B \rightarrow K \gamma \gamma) \sim\left[\frac{\left|\kappa_{c} Q_{u}^{2} C_{2}\right|^{2}}{\left|C_{9}\right|^{2}+\left|C_{10}\right|^{2}} \text { or } \frac{\left|C_{7}\right|^{2}}{\left|C_{9}\right|^{2}+\left|C_{10}\right|^{2}}\right] \times \mathscr{B}\left(B \rightarrow K \ell^{+} \ell^{-}\right) \simeq \mathscr{O}\left(10^{-9}\right) .
$$

The double photon decays are substantially suppressed with respect to the semileptonic ones, since the di-lepton operators with large coefficients $\left|C_{9,10}^{S M}\right| \simeq \mathscr{O}(4-5)$ are not contributing to the former.

To be comparable to the resonance contributions at least in some region of phase space the SD contribution has to be lifted by roughly one order of magnitude above the SM one. At the same time, such type of NP should not violate other data. A sizeable enhancement of the photon dipole coupling, $C_{7}$, is excluded by data on $B \rightarrow X_{s} \gamma$, which forces $\left|C_{7}\right| \simeq\left|C_{7}^{S M}\right|$. More room for NP is in the 4-Fermi operators, which contribute at higher order to $b \rightarrow s \gamma$ decays. A possibility are large non-standard (pseudo)scalar couplings to taus $\mathscr{O}_{S(P)}^{\tau}=\frac{\alpha_{e m}}{4 \pi} \bar{s} R b \bar{\tau}\left(\gamma_{5}\right) \tau$. From $\mathscr{B}\left(B_{s} \rightarrow \tau^{+} \tau^{-}\right)<5 \%$ [10] one obtains $\left|C_{S(P)}^{\tau}\right| \lesssim 700$, which is also consistent with data on other rare decays such as $b \rightarrow s \gamma$ and $b \rightarrow s \ell^{+} \ell^{-}, \ell=e, \mu$. In particular, large couplings are allowed because FCNC decays into a tau pair are essentially unconstrained to date. In other words, decays such as $B_{s} \rightarrow \tau^{+} \tau^{-}$and $B \rightarrow$ $K^{(*)} \tau^{+} \tau^{-}$have sizable room for NP. As can be seen from Fig.2, the corresponding maximal SD $B \rightarrow K \gamma \gamma$ spectrum with NP in the $\tau$-couplings leaks marginally outside the resonance background.

Having a close look at the exclusive $B \rightarrow K \gamma \gamma$ decays, using the HQET and the SCET formalisms, we found that the resulting SD branching ratio in the SM is small, order $10^{-9}$, in agree- 


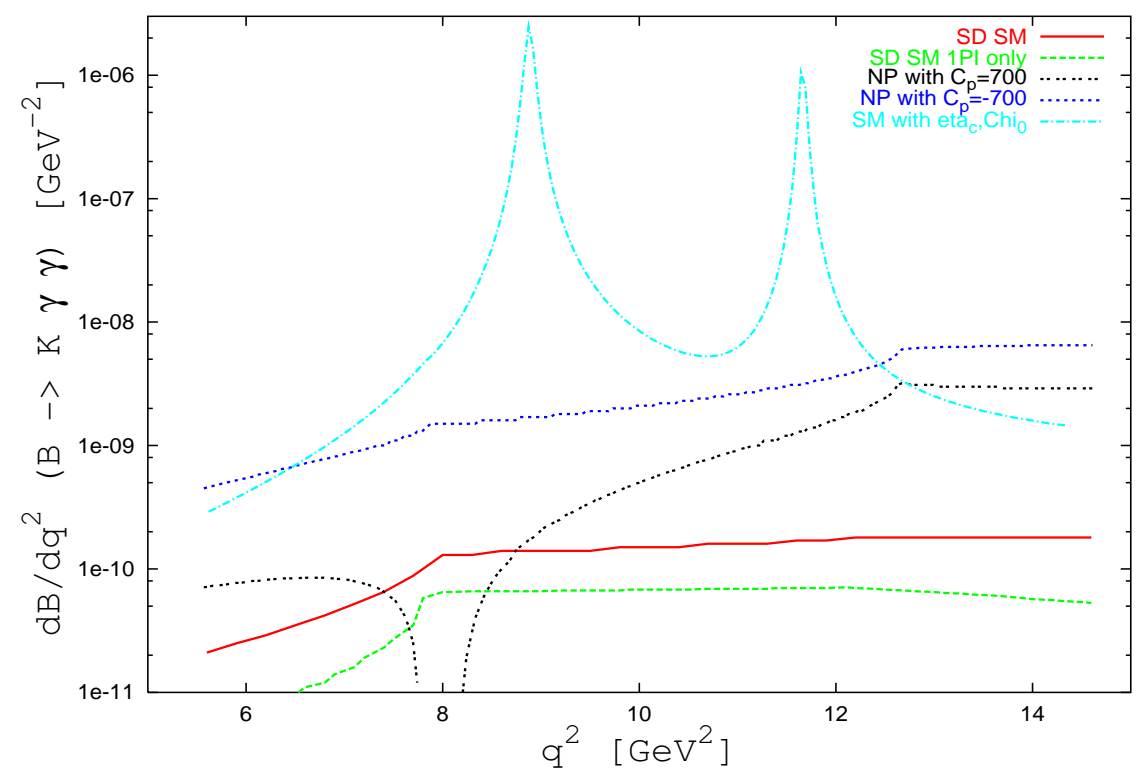

Figure 2: Di-photon mass distribution of $B \rightarrow K \gamma \gamma$ decays in the OPE region. The solid (dashed) curve denote the SM pure SD contribution with (and without) the 1PR terms. The dotted (double-dotted) curve corresponds to a NP scenario with $C_{P}^{\tau}=-700(+700)$ and $C_{S}^{\tau}=0$. The dash-dotted curve is the SM including the resonance contributions from the $\eta_{c}$ and the $\chi_{c 0}$. Figure taken from [1].

ment with the expectations based on the semileptonic rare $B \rightarrow K \ell^{+} \ell^{-}$decays. We discussed further contributions, including long-distance effects via $B \rightarrow \eta_{c} K \rightarrow \gamma \gamma K$ and $B \rightarrow \chi_{c 0} K \rightarrow \gamma \gamma K$. Unfortunately, we found that the SM SD contribution is not accessible behind the resonance peaks. We have further explored the possibility of NP contributions in $B \rightarrow K \gamma \gamma$. In particular, enhanced FCNC couplings to taus can give via the 1PI-loop branching ratios up to $\sim 10^{-8}$. The corresponding maximal SD di-photon spectrum is then for some range of $q^{2}$ above the resonance background. We conclude that it is difficult to test SD physics with exclusive $B \rightarrow K \gamma \gamma$ decays. However, even strongly resonance polluted, this mode could be useful to probe the photon helicity in $b \rightarrow s \gamma$ via interference of different resonance amplitudes, as pointed out recently in [11].

\section{References}

[1] G. Hiller and A. S. Safir, JHEP 0502 (2005) 011.

[2] L. Reina, G. Ricciardi and A. Soni, Phys. Rev. D 56, 5805 (1997).

[3] P. Singer and D. X. Zhang, Phys. Rev. D 56, 4274 (1997).

[4] S. R. Choudhury, G. C. Joshi, N. Mahajan and B. H. J. McKellar, Phys. Rev. D 67, 074016 (2003).

[5] C. W. Bauer, S. Fleming, D. Pirjol and I. W. Stewart, Phys. Rev. D 63, 114020 (2001).

[6] M. Beneke, A. P. Chapovsky, M. Diehl and T. Feldmann, Nucl. Phys. B 643, 431 (2002).

[7] J. Charles, A. Le Yaouanc, L. Oliver, O. Pène and J. C. Raynal, Phys. Rev. D 60, 014001 (1999).

[8] M. Beneke and T. Feldmann, Nucl. Phys. B 592, 3 (2001).

[9] A. Ali, P. Ball, L. T. Handoko and G. Hiller, Phys. Rev. D 61, 074024 (2000).

[10] Y. Grossman, Z. Ligeti and E. Nardi, Phys. Rev. D 55, 2768 (1997).

[11] M. Knecht and T. Schietinger, hep-ph/0509030. 